\title{
Liquid Biopsy for the Management of Patients with Colorectal Cancer
}

\author{
Takeshi Yamada Akihisa Matsuda Michihiro Koizumi Seiichi Shinji \\ Goro Takahashi Takuma Iwai Kohki Takeda Kohji Ueda Yasuyuki Yokoyama \\ Keisuke Hara Masahiro Hotta Satoshi Matsumoto Hiroshi Yoshida \\ Department of Gastrointestinal and Hepato-Biliary-Pancreatic Surgery, Nippon Medical School, Tokyo, Japan
}

\section{Keywords}

Circulating tumor DNA · Circulating tumor cell .

Heterogeneity $\cdot$ Minimal residual disease

\begin{abstract}
Background: Liquid biopsy is a collective term that refers to the analysis of tumor-derived biomarkers isolated from biological fluids of cancer patients. Recently, many authors reported the usefulness of liquid biopsy for the management of malignancy. Summary and Key Messages: The peripheral blood of cancer patients is a pool of cells and/or cell products derived from the primary or metastatic tumor, including circulating tumor cells (CTCs), circulating free (cf) DNA or RNA, and exosomes containing proteins, nucleic acids, and lipids. CTCs are tumor cells that can be isolated from peripheral blood. Free circulating DNA with a tumor-specific mutation is called circulating tumor DNA (ctDNA). Some patients who undergo curative surgery experience recurrent disease, which can be due to the presence of minimal residual disease (MRD). Thus, MRD indicates a high risk of relapse. Detection of ctDNA or CTC after surgery is a direct proof of MRD. Molecular volume (e.g., the number of CTCs and level of ctDNA) might reflect tumor burden, thus high molecular vol-
\end{abstract}

ume may indicate poor prognosis. The most notable application of liquid biopsy in cancer is to understand spatial and temporal heterogeneities. Heterogeneity is one of the causes of refractoriness and hampers prediction of chemotherapeutic effect. Emerging mutations that are not present in primary tumors but are found in their metastases can be detected in ctDNA. Some colorectal cancer patients with wildtype RAS do not respond to epidermal growth factor receptor blockade. In a subset of these patients, RAS mutation is detected in ctDNA, indicating heterogeneity.

C 2018 S. Karger AG, Basel

\section{Introduction}

The term liquid biopsy was originally introduced in relation to the analysis of circulating tumor cells (CTCs) [1]. Liquid biopsy is now a collective term that refers to the analysis of tumor-derived biomarkers isolated from biological fluids of cancer patients. Although peripheral blood is the main source of liquid biopsy, urine, pleural effusion, ascites, and cerebrospinal fluid are also used. The peripheral blood of a cancer patient contains a pool of cells and/or cell products derived from the primary or

\section{KARGER}

(c) 2018 S. Karger AG, Basel

E-Mail karger@karger.com

www.karger.com/dig
Takeshi Yamada, MD, PhD

Department of Digestive Surgery

Nippon Medical School, 1-1-5 Sendagi

Bunkyo-ku, Tokyo 113-8603 (Japan)

E-Maily-tak@nms.ac.jp 
metastatic tumor, including CTCs, circulating free (cf) DNA or RNA, and exosomes [2]. Analysis of these blood components can provide a comprehensive real-time picture of the tumor-associated changes in an individual cancer patient. These data can be used for the early detection of cancer, prognostic information (estimation of the risk for metastatic relapse or progression), predictive information (sensitivity to anticancer agents), monitoring of treatment response, and identification of minimal residual disease (MRD) [3]. Information from liquid biopsy is also useful for understanding the mechanisms of drug resistance, tumor progression, and metastasis.

Liquid biopsy has several advantages compared with conventional tissue biopsy, including a minimally invasive procedure, fast turnaround time, and the ability to provide a more comprehensive molecular landscape of the disease. The low invasiveness enables serial biopsy, and the fast turnaround time enables real-time monitoring with minimal time lag. The most notable application of liquid biopsy is to understand heterogeneity, which is often underestimated from a single tumor biopsy [4]. Heterogeneity is one of the major reasons for the refractory nature of cancer and can contribute to treatment failure and drug resistance. Heterogeneity includes spatial heterogeneity and temporal heterogeneity. Spatial heterogeneity refers to intratumor heterogeneity and intertumor heterogeneity between primary and metastatic tumor or between metastatic tumors. Temporal heterogeneity refers to genetic profile alterations that are mainly caused by anticancer agents.

In this study, we will provide an overview of the current status of the field of liquid biopsy and its role in selection of treatment and the management of colorectal cancer (CRC). We will mainly focus our discussion on prognostic and predictive information provided by liquid biopsy.

\section{Types of Liquid Biopsy}

Contamination is a major problem in liquid biopsy. It is hard to capture CTCs exclusively because most systems also capture a lot of leukocytes. One milliliter of blood includes approximately 1 CTC and $10^{7}$ leukocytes. It is also hard to distinguish DNA fragments released by the tumor from those released by normal cells. The ratio of circulating free DNA (cfDNA) released by cancer cells to total cfDNA is highly variable, ranging between $0.01 \%$ and greater than $90 \%$.
Analysis of both CTCs and ctDNA can provide qualitative (presence or absence of mutation) and quantitative (e.g., the number of CTCs and level of ctDNA) information. RNA and protein cannot be analyzed using cfDNA alone. In contrast, CTC analysis can provide various information including DNA, RNA, and protein although the amount of DNA in CTCs is much smaller than the amount of ctDNA harvested from the same volume of peripheral blood. Therefore, the accuracy of mutation detection using cfDNA is better than that using CTCs.

\section{Circulating Tumor Cells}

CTCs are tumor cells that can be isolated from peripheral blood. Solid tumors can release a surprisingly high number of CTCs every day into the circulation. However, most of these cells die in the circulation as a result of mechanical and environmental trauma such as shear forces, oxidative stress, and attack by the immune system, and the half-life of CTCs in the bloodstream is only $1-2.4 \mathrm{~h}$ [5]. Investigation of CTCs might allow comprehensive studies at the DNA, RNA, and protein levels; however, their heterogeneity and scarcity are obstacles to obtaining a deep understanding of these cells. The number of CTCs is generally low in patients with CRC [6], limiting the possibility of clinical applications of CTC analysis despite the development of many different methods. DNA analysis requires 1 ng of DNA, whereas 10 or fewer CTCs (one cell contains 6 pg of DNA) are typically harvested using the methods that have been developed to date.

One of the reasons why massive amounts of CTCs cannot be captured might be the epithelial-mesenchymal transition (EMT), a process linked to cancer stemness and chemotherapy resistance. CTCs express various EMT-related transcription factors (e.g., Twist and Snail), fibronectin, and vimentin which is a typical mesenchymal intermediate filament protein [7]. In most techniques, the first step of the CTC isolation technique involves enrichment, which is followed by a capture step. CTC enrichment techniques are based on biological (e.g., immunoselection) or physical (e.g., size, density, deformability, electrical charge) distinctions. Immunoselection is based on the expression of specific surface markers, in most cases epithelial cell adhesion molecule. Leukocytes are negatively selected using leukocytic antigens such as CD45. More sensitive technologies that can capture CTCs that have undergone EMT are currently being developed.
Yamada et al. 
CTCs can be used to build in vitro and in vivo experimental models, which can provide an immense amount of genetic and epigenetic information on tumor biology and assess the sensitivity to anticancer drugs [8].

\section{Circulating Free DNA and Circulating Tumor DNA}

cfDNA exists in peripheral blood, urine, ascites, and pleural effusion. cfDNA that possesses a tumor-specific mutation is called circulating tumor DNA (ctDNA). At present there is no way to specifically isolate ctDNA from other circulating DNAs, and only the detection of tumorspecific mutations on cfDNA indicates the presence of ctDNA. ctDNA fragments mainly originate from apoptotic or necrotic tumor cells [9]. Normal DNA released from dying blood cells after blood collection contaminates and dilutes ctDNA. In healthy individuals, the main source of cfDNA is apoptotic cells derived from the hematological system, the gastrointestinal tract, and the skin, but predominantly from white blood cells. In cancer patients, both apoptotic and necrotic cells are a major source of cfDNA [10]. cfDNA concentrations are higher in CRC patients than in healthy individuals $[11,12]$. In addition, the proportion of cfDNA fragments smaller than 100 bp is greater in CRC patients than in healthy individuals. Conversely, the proportion of cfDNA fragments larger than $300 \mathrm{bp}$ is greater in healthy individuals than in CRC patients [12].

\section{MicroRNA}

Exosomes (40-150 $\mathrm{nm}$ in diameter) originate from secretory multivesicular bodies that fuse with the plasma membrane resulting in the release of vesicles into the extracellular space. Exosomes contain proteins, nucleic acids, and lipids and can represent a better source of nucleic acids for tumor molecular profiling compared with cell-free nucleic acids, particularly with regard to mRNA that is sensitive to RNAse degradation.

\section{What Information Can Liquid Biopsy Provide to Gastroenterologists?}

In cases of potentially curative disease, liquid biopsy can provide information about the risk of relapse and prognosis by detecting MRD. In metastatic disease, liquid biopsy can provide information about prognosis by measuring the tumor volume and can help in the prediction of tumor sensitivity to targeted therapies by detecting mutation of the target or by monitoring the change of tumor volume.

\section{Minimal Residual Disease}

The majority of localized cancers can be cured by surgery alone without any systemic therapy. However, some patients who undergo curative surgery experience recurrent disease. Identifying the patients at high risk of relapse, and treating them with adjuvant therapy, remains a major clinical issue. A small subset of stage I patients and a larger subset of stage II patients relapse and could potentially benefit from adjuvant therapy whereas some stage III patients do not need adjuvant therapy. The reason why some patients experience recurrent disease might be due to the presence of MRD. The biomarker currently used as the standard of care, carcinoembryonic antigen, and clinicopathological factors have limited sensitivity and specificity for MRD.

In theory, at the time of diagnosis of disease the number of CTCs and/or the ctDNA level is high. After curative surgery, CTC and ctDNA disappear immediately from the peripheral blood because both have a rapid turnaround time in the order of a few hours. However, CTC and ctDNA can be detected in patients with MRD, and clinical recurrence can be detected by diagnostic imaging after their increase. Some patients without MRD experience recurrent disease; however, the time to recurrence is longer than that of patients with MRD (Fig. 1).

However, there is limited evidence demonstrating these phenomena. Postoperative detection of ctDNA provides evidence of MRD and identifies patients at very high risk of relapse among CRC patients who undergo curative surgery [13]. Also, stage II CRC patients with MRD detected using ctDNA had a very high risk of recurrence. In this study, recurrence occurred in $79 \%$ of patients with positive ctDNA after surgery, conversely, recurrence occurred in only $9.8 \%$ of patients with negative ctDNA [14]. The presence of CTCs after curative surgery was associated with progression-free survival and overall survival (OS). In this study, recurrence occurred in $72.5 \%$ of patients with positive CTCs after surgery, conversely, recurrence occurred in only $12.2 \%$ of patients with negative CTCs [15]. 
Fig. 1. Overview of the potential clinical applications of ctDNA and CTCs depending on the clinical setting. At the time of diagnosis of disease, the number of CTCs and/ or ctDNA level was high. After curative surgery, CTCs and ctDNA disappear immediately from the peripheral blood. However, CTCs and ctDNA can be detected in MRD and an increase in their levels indicates clinical recurrence. Although some patients without MRD can experience recurrent disease, the time to recurrence is longer than that of patients with MRD. ctDNA, circulating tumor DNA; CTCs, circulating tumor cells; MRD, minimal residual disease.

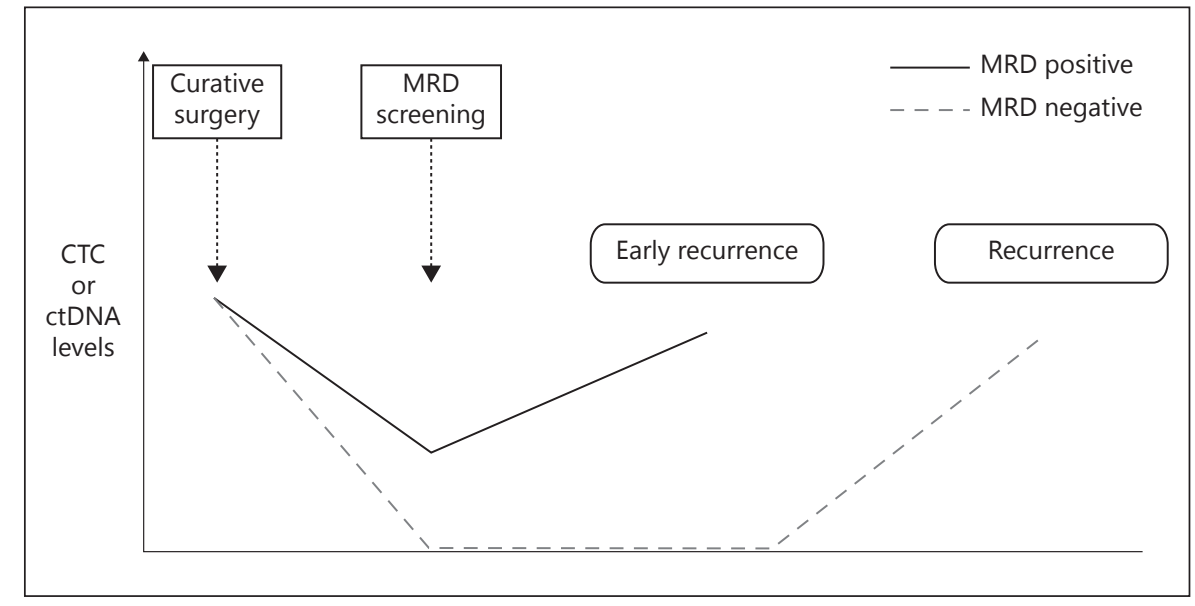

\section{Prognostic Information for Metastatic Disease}

The presence of CTCs, which can cause metastases, is itself a prognostic marker in patients with CRC. A metaanalysis showed that the hazard ratio of recurrence-free survival was 3.06 and that of OS was 2.70 [16]. As CTCs are shed from the primary tumor and/or metastatic lesions into the vasculature, the presence of CTCs does not always indicate the presence of metastasis. However, CTCs are considered to be seeds distributed by primary tumors for the potential initiation of metastatic growth at distant organ sites. In metastatic disease, the number of CTCs is strongly linked to cancer progression and treatment response, and a high number of CTCs is associated with shorter progression-free survival and OS.

Molecular volume (e.g., the number of CTCs and level of ctDNA) might reflect tumor burden in a similar manner to tumor volume calculated by diagnostic imaging (e.g., CT or MRI). Response Evaluation Criteria in Solid Tumors was developed as an easy method to evaluate the tumor burden; however, it does not always indicate true tumor burden and prognosis. High molecular volume might indicate high tumor burden and an increase in molecular volume might indicate an increasing tumor burden, which can cause poor prognosis.

Several cf microRNAs have been reported as prognostic biomarkers. Levels of circulating miR-21 are elevated in patients with CRC, circulating miR-21 decreased in postoperative serum from patients who underwent curative surgery, and high miR-21 expression is associated with poor prognosis [17]. High circulating miR-200c in serum demonstrates a significant positive correlation with lymph node and distant metastasis and prognosis [18]. Circulating miR-1290 levels in serum are signifi- cantly upregulated in patients with CRCs and high miR1290 expression in serum is associated with poor prognosis. High miR-1290 expression is an independent predictor for tumor recurrence [19]. High levels of circulating miR-203 in serum are associated with poor survival and metastasis [20].

\section{Predictive Information for Metastatic Disease}

Both spatial and temporal heterogeneity hamper prediction of the effect of molecular target therapy. Emerging mutations that are not present in primary tumors may be found in their metastases and can be detected in ctDNA [21]. Direct analysis of metastatic cells reveal important information for systemic cancer therapy targeting metastatic disease. As different metastatic sites can harbor different genomic aberrations, conventional biopsy of 1 or 2 accessible metastases may not be representative [4].

At the time of selection of a molecular targeted drug, liquid biopsy can provide molecular information for the metastatic tumor. After initiation of treatment, changes in molecular volume (e.g., the number of CTCs or level of ctDNA) can be useful for the prediction and monitoring of the response. Temporal heterogeneity detected by liquid biopsy is a good predictor of secondary resistance. Liquid biopsy also provides molecular information at the time of selection of second-line chemotherapy. In most cases, molecular information from the liquid biopsy differs from that of the primary tumor or the results of liquid biopsy performed before starting first-line chemotherapy. Thus, serial liquid biopsy can help in the management of CRC patients under chemotherapy (Fig. 2).
Yamada et al. 


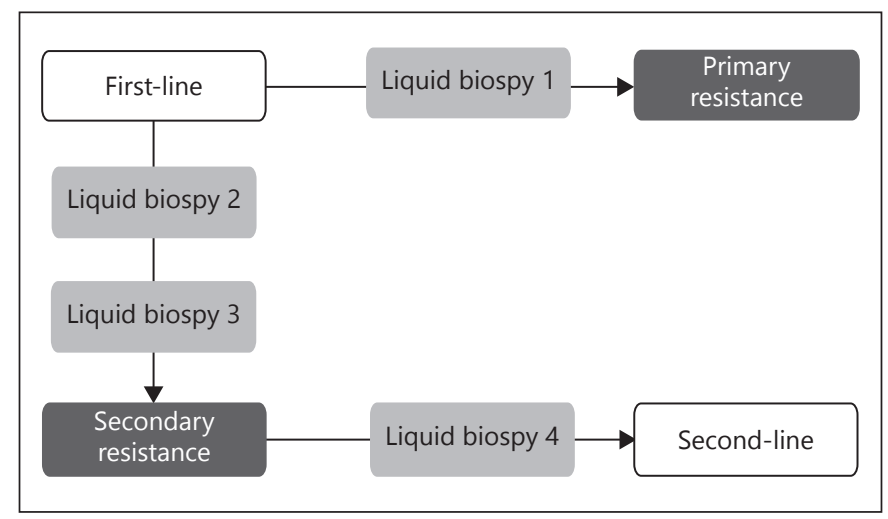

Fig. 2. Treatment selection and monitoring using liquid biopsy. At the time of selection of molecular targeted drugs liquid biopsy provides information about spatial heterogeneity, which is a good predictor of primary resistance. In some patients whose primary tumor has no RAS mutation, RAS mutation is detected in liquid biopsy. The molecular volume (e.g., number of circulating tumor cells or levels of circulating tumor DNA) before starting chemotherapy is a prognostic biomarker (Liquid biopsy 1). After initiation of treatment, changes in molecular volume can be useful for the prediction and monitoring of the response. In patients with early tumor shrinkage, molecular volume decreases 4 weeks after starting chemotherapy (Liquid biopsy 2). Temporal heterogeneity detected by liquid biopsy is a good predictor of secondary resistance. Emerging KRAS mutation are detected in cfDNA from patients with secondary resistance to epidermal growth factor receptor (EGFR) blockade. In addition, molecular volume increase after getting acquired resistance (Liquid biopsy 3 ). Regarding selection of second-line chemotherapy, in many cases the molecular information provided from the liquid biopsy differs from that of primary tumor or the results of liquid biopsy before starting first-line chemotherapy (Liquid biopsy 4).

CTCs have the potential to provide predictive information about the effect of molecular targeted therapy; however, their scarcity limits this potential. The detection rate of KRAS mutation using ctDNA is much higher than that using CTCs. miRNA also provides limited predictive information about the effect of chemotherapy.

Some CRC patients with wild-type RAS do not respond to epidermal growth factor receptor (EGFR) blockade. One explanation for this failure could be heterogeneity between primary and metastatic tumors [22]. We previously reported the detection of KRAS mutations in cfDNA from 5 of $55 \mathrm{mCRC}$ patients (9\%) with wild-type $K R A S$ in their primary tumors [23]. Other researchers reported that KRAS mutation in cfDNA is a negative biomarker of EGFR blockade chemotherapy [23-27].

Temporal heterogeneity is one of the causes of acquired resistance. Some researchers have detected emerging KRAS mutation in cfDNA from patients with second- ary resistance to EGFR blockade chemotherapy [24-26, 28-30]. Emerging BRAF mutation [25] and emerging EGFR mutation $[26,30]$ are induced by EGFR blockade.

\section{Future Perspectives}

Despite the many clinical studies on liquid biopsy conducted in CRC and the promising preliminary results, the use of this approach in clinical practice is still extremely limited. The reasons for this include disparity in methods, the limited number of patients enrolled, and the lack of a clear demonstration of a clinical benefit. However, liquid biopsy is an absolutely attractive tool, and has 3 exclusive strengths: the ability to detect spatial and temporal heterogeneities, the ability to detect MRD, and the ability to monitor molecular volume change.

Detection of spatial and temporal heterogeneities is critical for determining the indication of molecular target therapy. At the present time, the ability to detect mutations using ctDNA is superior to that using CTCs; however, the value ofCTCs might improve if massive amounts of CTCs can be captured. Tumor educated platelets are also candidates for liquid biopsy.

Minimal residual disease could replace TMN classification as a method of judging the need for adjuvant therapy. Lymph node metastasis only indicates the possibility of MRD; in other words, it provides indirect proof of MRD. In contrast, the presence of ctDNA or CTC after surgery is a direct proof of MRD.

Molecular volume could replace the Response Evaluation Criteria in Solid Tumors as a way to monitor the effect of chemotherapy. Measure of molecular volume does not involve radiation exposure. Moreover, the fast turnover of ctDNA and CTC enables early diagnosis of a response. This approach is of particular benefit in cancer, which has no available tumor markers such as cholangiocellular carcinoma [31].

\section{Conclusion}

Peripheral blood of cancer patients is an attractive source of much useful information. Liquid biopsy involves various targets including CTCs, ctDNA, and miRNA. Serial liquid biopsy provides qualitative and quantitative information that is useful for judging the risk of relapse by detecting MRD, for treatment selection by revealing spatial and temporal heterogeneities, and for prognostication by measuring the molecular volume of the tumor. 


\section{Acknowledgments}

We thank Mary Derry, PhD ELS, from Edanz Group (www. edanzediting.com/ac) for editing a draft of this manuscript.

\section{Disclosure Statement}

The authors have no potential conflicts of interest to declare.

\section{References}

1 Alix-Panabieres C: Circulating tumor cells: liquid biopsy of cancer. Clin Chem 2013;59: $110-118$.

2 Joosse SA, Pantel K: Tumor-educated platelets as liquid biopsy in cancer patients. Cancer Cell 2015;28:552-554.

3 Cohen JD, Li L, Wang Y, Thoburn C, Afsari B, Danilova L, Douville C, Javed AA, Wong F, Mattox A, Hruban RH, Wolfgang CL, Goggins MG, Dal Molin M, Wang TL, Roden R, Klein AP, Ptak J, Dobbyn L, Schaefer J, Silliman N, Popoli M, Vogelstein JT, Browne JD, Schoen RE, Brand RE, Tie J, Gibbs P, Wong HL, Mansfield AS, Jen J, Hanash SM, Falconi M, Allen PJ, Zhou S, Bettegowda C, Diaz LA Jr, Tomasetti C, Kinzler KW, Vogelstein B, Lennon AM, Papadopoulos N: Detection and localization of surgically resectable cancers with a multi-analyte blood test. Science 2018; 359:926-930.

4 Gerlinger M, Rowan AJ, Horswell S, Math M, Larkin J, Endesfelder D, Gronroos E, Martinez P, Matthews N, Stewart A, Tarpey P, Varela I, Phillimore B, Begum S, McDonald NQ, Butler A, Jones D, Raine K, Latimer C, Santos CR, Nohadani M, Eklund AC, Spencer-Dene B, Clark G, Pickering L, Stamp G, Gore M, Szallasi Z, Downward J, Futreal PA, Swanton C: Intratumor heterogeneity and branched evolution revealed by multiregion sequencing. New Eng J Med 2012;366:883892.

5 Meng S, Tripathy D, Frenkel EP, Shete S, Naftalis EZ, Huth JF, Beitsch PD, Leitch M, Hoover S, Euhus D, Haley B, Morrison L, Fleming TP, Herlyn D, Terstappen LW, Fehm T, Tucker TF, Lane N, Wang J, Uhr JW: Circulating tumor cells in patients with breast cancer dormancy. Clin Cancer Res 2004;10: 8152-8162.

6 Cohen SJ, Punt CJ, Iannotti N, Saidman BH, Sabbath KD, Gabrail NY, Picus J, Morse MA, Mitchell E, Miller MC, Doyle GV, Tissing H, Terstappen LW, Meropol NJ: Prognostic significance of circulating tumor cells in patients with metastatic colorectal cancer. Ann Oncol 2009;20:1223-1229.

7 Pantel K, Alix-Panabieres C: Real-time liquid biopsy in cancer patients: fact or fiction? Cancer Res 2013;73:6384-6388.

8 Cayrefourcq L, Mazard T, Joosse S, Solassol J, Ramos J, Assenat E, Schumacher U, Costes V, Maudelonde T, Pantel K, AlixPanabieres C: Establishment and characterization of a cell line from human circulating colon cancer cells. Cancer Res 2015;75:892901.
9 Schwarzenbach H, Hoon DS, Pantel K: Cellfree nucleic acids as biomarkers in cancer patients. Nature Rev Cancer 2011;11:426-437.

10 Jahr S, Hentze H, Englisch S, Hardt D, Fackelmayer FO, Hesch RD, Knippers R: DNA fragments in the blood plasma of cancer patients: quantitations and evidence for their origin from apoptotic and necrotic cells. Cancer Res 2001;61:1659-1665.

11 Spindler KL, Appelt AL, Pallisgaard N, Andersen RF, Brandslund I, Jakobsen A: Cellfree DNA in healthy individuals, noncancerous disease and strong prognostic value in colorectal cancer. Int J Cancer 2014;135: 2984-2991.

12 Mouliere F, El Messaoudi S, Pang D, Dritschilo A, Thierry AR: Multi-marker analysis of circulating cell-free DNA toward personalized medicine for colorectal cancer. Mol Oncol 2014;8:927-941.

13 Scholer LV, Reinert T, Orntoft MW, Kassentoft CG, Arnadottir SS, Vang S, Nordentoft I, Knudsen M, LamyP, Andreasen D, Mortensen FV, Knudsen AR, Stribolt K, Sivesgaard K, Mouritzen P, Nielsen HJ, Laurberg S, Orntoft $\mathrm{TF}$, Andersen CL: Clinical implications of monitoring circulating tumor DNA in patients with colorectal cancer. Clin Cancer Res 2017;23:5437-5445.

14 Tie J, Wang Y, Tomasetti C, Li L, Springer S, Kinde I, Silliman N, Tacey M, Wong HL, Christie M, Kosmider S, Skinner I, Wong R, Steel M, Tran B, Desai J, Jones I, Haydon A, Hayes T, Price TJ, Strausberg RL, Diaz LA Jr, Papadopoulos N, Kinzler KW, Vogelstein B, Gibbs P: Circulating tumor DNA analysis detects minimal residual disease and predicts recurrence in patients with stage II colon cancer. Science Transl Med 2016;8: 346 ra392.

15 Lu CY, Uen YH, Tsai HL, Chuang SC, Hou MF, Wu DC, Juo SH, Lin SR, Wang JY: Molecular detection of persistent postoperative circulating tumour cells in stages II and III colon cancer patients via multiple blood sampling: prognostic significance of detection for early relapse. Br J Cancer 2011;104:11781184 .

16 Rahbari NN, Aigner M, Thorlund K, Mollberg N, Motschall E, Jensen K, Diener MK, Buchler MW, Koch M, Weitz J: Meta-analysis shows that detection of circulating tumor cells indicates poor prognosis in patients with colorectal cancer. Gastroenterology 2010;138: 1714-1726.

17 Toiyama Y, Takahashi M, Hur K, Nagasaka T, Tanaka K, Inoue Y, Kusunoki M, Boland CR,
Goel A: Serum miR-21 as a diagnostic and prognostic biomarker in colorectal cancer. J Nat Cancer Inst 2013;105:849-859.

18 Toiyama Y, Hur K, Tanaka K, Inoue Y, Kusunoki M, Boland CR, Goel A: Serum miR-200c is a novel prognostic and metastasis-predictive biomarker in patients with colorectal cancer. Ann Surg 2014;259:735-743.

19 Imaoka H, Toiyama Y, Fujikawa H, Hiro J, Saigusa S, Tanaka K, Inoue Y, Mohri Y, Mori T, Kato T, Toden S, Goel A, Kusunoki M: Circulating microRNA-1290 as a novel diagnostic and prognostic biomarker in human colorectal cancer. Ann Oncology 2016;27: 1879-1886.

20 Hur K, Toiyama Y, Okugawa Y, Ide S, Imaoka $\mathrm{H}$, Boland CR, Goel A: Circulating microRNA-203 predicts prognosis and metastasis in human colorectal cancer. Gut 2017;66:654665 .

21 Furuki H, Yamada T, Takahashi G, Iwai T, Koizumi M, Shinji S, Yokoyama Y, Takeda K, Taniai N, Uchida E: Evaluation of liquid biopsies for detection of emerging mutated genes in metastatic colorectal cancer. Eur J Surg Oncol 2018;44:975-982.

22 Baldus SE, Schaefer KL, Engers R, Hartleb D, Stoecklein NH, Gabbert HE: Prevalence and heterogeneity of KRAS, BRAF, and PIK3CA mutations in primary colorectal adenocarcinomas and their corresponding metastases. Clin Cancer Res 2010;16:790799

23 Yamada T, Iwai T, Takahashi G, Kan H, Koizumi M, Matsuda A, Shinji S, Yamagishi A, Yokoyama Y, Tatsuguchi A, Kawagoe T, Kitano S, Nakayama M, Matsumoto S, Uchida E: Utility of KRAS mutation detection using circulating cell-free DNA from patients with colorectal cancer. Cancer Sci 2016;107:936943.

24 Vidal J, Muinelo L, Dalmases A, Jones F, Edelstein D, Iglesias M, Orrillo M, Abalo A, Rodríguez C, Brozos E, Vidal Y, Candamio S, Vázquez F, Ruiz J, Guix M, Visa L, Sikri V, Albanell J, Bellosillo B, López R, Montagut C: Plasma ctDNA RAS mutation analysis for the diagnosis and treatment monitoring of metastatic colorectal cancer patients. Ann Oncol 2017;28:1325-1332.

25 Spindler KL, Pallisgaard N, Andersen RF, Jakobsen A: Changes in mutational status during third-line treatment for metastatic colorectal cancer - results of consecutive measurement of cell free DNA, KRAS and BRAF in the plasma. Int J Cancer 2014;135: 2215-2222. 
26 Morelli MP, Overman MJ, Dasari A, Kazmi SM, Mazard T, Vilar E, Morris VK, Lee MS, Herron D, Eng C, Morris J, Kee BK, Janku F, Deaton FL, Garrett C, Maru D, Diehl F, Angenendt $\mathrm{P}$, Kopetz S: Characterizing the patterns of clonal selection in circulating tumor DNA from patients with colorectal cancer refractory to anti-EGFR treatment. Ann Oncol 2015;26:731-736.

27 Grasselli J, Elez E, Caratù G, Matito J, Santos C, Macarulla T, Vidal J, Garcia M, Viéitez JM, Paéz D, Falcó E, Lopez Lopez C, Aranda E, Jones F, Sikri V, Nuciforo P, Fasani R, Tabernero J, Montagut C, Azuara D, Dienstmann R, Salazar R, Vivancos A: Concordance of blood- and tumor-based detection of RAS mutations to guide anti-EGFR therapy in metastatic colorectal cancer. Ann Oncol 2017; 28:1294-1301.

28 Misale S, Yaeger R, Hobor S, Scala E, Janakiraman $\mathrm{M}$, Liska D, Valtorta E, Schiavo R, Buscarino M, Siravegna G, Bencardino K, Cercek A, Chen CT, Veronese S, Zanon C, Sartore-Bianchi A, Gambacorta M, Gallicchio M, Vakiani E, Boscaro V, Medico E, Weiser M, Siena S, Di Nicolantonio F, Solit D, Bardelli A: Emergence of KRAS mutations and acquired resistance to anti-EGFR therapy in colorectal cancer. Nature 2012;486: 532-536.

29 Diaz LA Jr, Williams RT, Wu J, Kinde I, Hecht JR, Berlin J, Allen B, Bozic I, Reiter JG, Nowak MA, Kinzler KW, Oliner KS, Vogelstein B: The molecular evolution of acquired resistance to targeted EGFR blockade in colorectal cancers. Nature 2012;486:537540

30 Siravegna G, Mussolin B, Buscarino M, Corti G, Cassingena A, Crisafulli G, Ponzetti A, Cremolini C, Amatu A, Lauricella C, Lamba S, Hobor S, Avallone A, Valtorta E, Rospo G, Medico E, Motta V, Antoniotti C, Tatangelo F, Bellosillo B, Veronese S, Budillon A, Montagut C, Racca P, Marsoni S, Falcone A, Corcoran RB, Di Nicolantonio F, Loupakis F, Siena S, Sartore-Bianchi A, Bardelli A: Clonal evolution and resistance to EGFR blockade in the blood of colorectal cancer patients. Nature Med 2015;21:795-801.

31 Vogel A, Saborowski A: Cholangiocellular carcinoma. Digestion 2017;95:181-185. 